\title{
In-use calibration of body-mounted gyroscopes for applications in gait analysis
}

\author{
Sergio Scapellato*, Filippo Cavallo, Chiara Martelloni, Angelo M. Sabatini \\ Scuola Superiore Sant'Anna, Piazza Martiri della Libertà 33, 56127 Pisa, Italy
}

Received 13 September 2004; received in revised form 22 February 2005; accepted 3 March 2005

Available online 10 May 2005

\begin{abstract}
In this paper, we propose an in-use calibration procedure for gyroscopes. The case report is a simple inertial measurement unit (IMU), which is used in our current research on inertial motion-sensing for advanced footware. The IMU contains two biaxial accelerometers and one gyroscope; it is developed for being mounted on one subject's foot instep, with the aim to reconstruct the trajectory in the sagittal plane of the sensed anatomical point. Since the IMU sagittal displacements can be estimated by performing strapdown integration, they can also be compared with their true values. One movement, which corresponds to known (vertical) displacements, consists of foot placements from the ground level on to top of steps of known height (step climbing). Provided that the IMU accelerometers are calibrated separately by any standard calibration procedure, motion tracking during the stepping movement allows to estimate the gyroscope sensitivity. The experimental results we present in this paper demonstrate the proposed in-use calibration procedure.
\end{abstract}

(C) 2005 Elsevier B.V. All rights reserved.

Keywords: Inertial measurement unit; Sensor calibration; Inertial motion-sensing; Gait analysis

\section{Introduction}

With the term inertial measurement units (IMU), it is customary to name devices, which embody inertial sensors - gyroscopes and/or accelerometers - mostly for being installed on man-made vehicles where navigation assistance is required, including spacecrafts, aircrafts and robots. The signals produced from these sensors - angular velocity and linear acceleration - can be processed to sense movement and orientation of the moving body where they are affixed [1].

Quite recently, inertial motion-sensing has found wide acceptance in other technological fields, including quantitative motion analysis as applied in biomedical and rehabilitation engineering. The main reason for this growing popularity is due to the opportunity they offer to sense motion and orienta-

\footnotetext{
* Corresponding author. Present address: Polo Sant'Anna Valdera, v. R. Piaggio 34, 56025, Pontedera (PI), Italy. Tel.: +39 050 883484; fax: +39050883497 .

E-mail address: sergio@arts.sssup.it (S. Scapellato).
}

tion without the restrictions, the encumbrance and the costs associated to the use of standard equipment existing within traditional motion analysis laboratories, e.g., video motionsensing.

IMU attached to the body of tested subjects in several anatomical positions (head, chest, trunk, thigh, shank and foot) can be used to reconstruct joint angle rotations, to estimate spatio-temporal parameters of gait, to perform sophisticated motion tracking functions, owing to their capability of reconstructing the trajectory of sensed anatomical points in the three-dimensional space [2]. Reported applications in the medical field are to monitor activities of daily living and to estimate the energy expenditure incurred during a functional activity [3], both in normal and pathological conditions.

One of the most critical aspects connected with the use of inertial motion-sensing is represented by the influence of sensor bias and sensitivity drifts on the accuracy of inertial processing; uncertainty in the values of bias and sensitivity of these devices, due to the influence of environmental conditions are time-integrated and propagates up to the level that 
the reconstructed trajectory is unreliable and inaccurate. For applications in the biomedical field, it is thus of the utmost importance the development of calibration procedures which can be used to check and verify the sensor offset and sensitivity during normal use of the IMU. Simple in-use calibration procedures for inertial sensors are described in [4], they are based on the possibility to move the sensor case in different orientations relative to the gravity field (accelerometric calibration) or to rotate the sensor case through known rotation angles (gyroscopic calibration) [7]. This possibility to manipulate the sensor case is precluded once the IMU is affixed to the body. A reasonable approach is then to instruct the subject to move, in order to get the information needed to verify sensor calibration. In Ref. [5], a simple procedure is described for achieving in-use calibration of triaxial accelerometers that are body-mounted. The procedure exploits the known fact that, in static conditions, the norm of the acceleration vector from a triaxial accelerometer would be $1 g\left(g=9.81 \mathrm{~m} / \mathrm{s}^{2}\right)$. The instructions are then to change postures and to let the system to collect the sensorial information needed for calibration in the new postures. Similar-in-concept in-use calibration procedures are not yet available for gyroscopes. In this paper, we propose a simple in-use calibration procedure for gyroscopes. The procedure is applied to the IMU for advanced footware we are currently working on. After accelerometers are calibrated by standard procedures, movements such as climbing a step of known height are performed. Provided that the strapdown integration is performed by combining accelerometer and gyroscope signals, the optimal value of the sensor sensitivity for which the estimated distance comes closer to the true distance, which is assumed to be known, is searched. The level of agreement between the proposed in-use calibration procedure and standard calibration procedures is analyzed and discussed in the experiments reported in this paper. During both procedures, we verified thermal stability to assure that temperature did not influence sensor output.

In the last section is then described a simple motion analysis experiment, the reconstruction of foot movements during stair climbing, to test results obtained through in-use calibration.

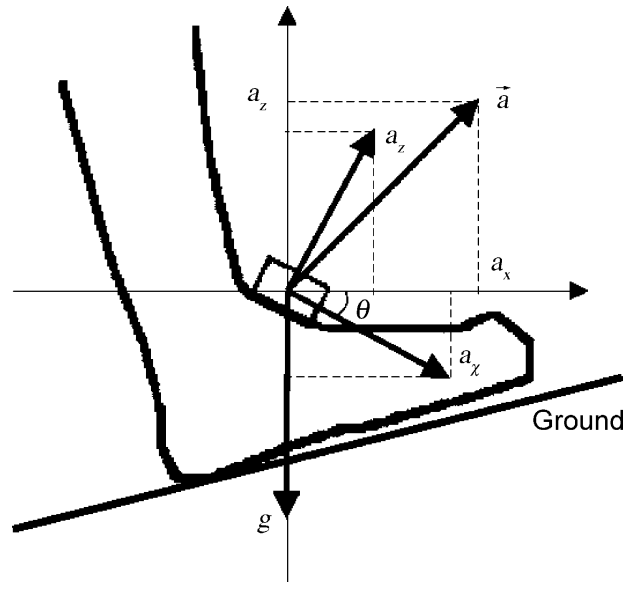

Fig. 2. IMU affixed to foot instep.

\section{Instruments}

The IMU (Fig. 1) for advanced footware sketched in was proposed to estimate a number of interesting gait parameters - stride time/length, cadence, walking speed and incline. It was composed of one gyroscope (Murata ENC-03J) and two biaxial accelerometers (Analog Devices ADXL210), arranged to form a triad of mutually perpendicular accelerometers; on IMU board are also integrated two simple driver circuits to interface sensor analog outputs: the accelerometric signal is sent directly to a buffer while the gyroscopic signal is sent to an analog filtering stage (accordingly with [6]). Both buffer and filtering circuits are made using a dual low-cost, rail-to-rail and single supply operational amplifier.

Once the IMU is affixed to the foot instep as shown in Fig. 2, the sensitive axis of the gyroscope is oriented in the medio-lateral direction.

The gyroscope measures the angular velocity around its sensitive axis, assumed to be orthogonal to sagittal plane, and allows to reconstruct IMU orientation in the sagittal plane $\theta$ by integration; the knowledge of $\theta$ is needed to project sensor output acceleration components $\left[a_{x}, a_{z}\right]$ over the sagittal directions $\left[a_{X}, a_{Z}\right]$ and also to remove gravitational contribution $g$.

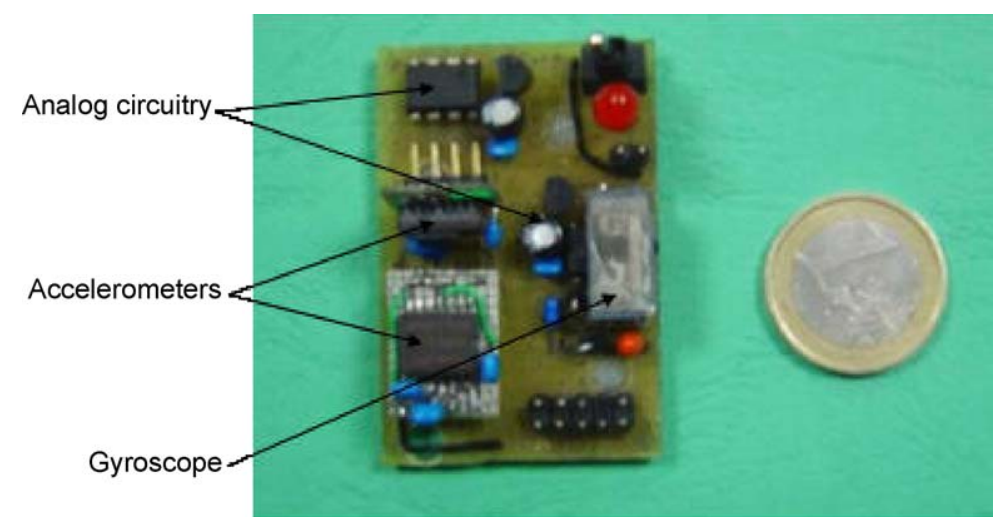

Fig. 1. IMU board containing two biaxial accelerometers, one gyroscope and a simple analog circuit. 
Before sampling, the accelerometer and gyroscope signals were single-stage low-pass filtered at $50 \mathrm{~Hz}$ and amplified. Twelve-bit sampling was performed at $f_{\mathrm{s}}=1 \mathrm{kHz}$ by using a PCMCIA card (National Instruments (NI) DAQ card AI-16E-4); this card was controlled by NI's LabView v. 6.1 software, for data acquisition and storage. Matlab v. 6.0 (The MathWorks) was used for off-line signal processing. A second-order forward-backward low-pass Butterworth filter was applied to sensor signals (cut-off frequency: $15 \mathrm{~Hz}$ ). Data decimation was also performed, in order to assess its influence on strapdown integration.

Together with IMU signals, we acquired also temperature data, at a sampling frequency of $1 \mathrm{~Hz}$, from an RTD sensor located on DAQ board; since this device is closed to IMU it can be assumed that measured temperature is the same of that on the foot. Temperature data were used to verify thermal stability during calibration tasks, because any significant variation of temperature brings to unreliable sensor calibration.

\section{Methods}

\subsection{Standard calibration procedure}

The standard calibration procedure for the triaxial accelerometer was based on its sensitivity to Earth gravitational field, the calibration was performed by placing the accelerometer sensitive axes in line with gravity, when the nominal output were $+1 g$ and $-1 g$.

The standard calibration procedure for the gyroscope consisted of imparting a known rotation $\left(+90^{\circ}\right)$ to the case containing the sensor; the gyroscope signal was then integrated to measure the angular excursion, then sensitivity was estimated from comparison between the estimated rotation angle and the "true" one.
Gyroscope's behaviour at varying temperature can be described with a simple first-order equation:

$V=\beta_{1} T+\beta_{0}+\varepsilon$

where $V$ is the gyroscope output voltage, $\beta_{0}$ and $\beta_{1}$ the regression coefficients of first-order equation, $T$ the temperature on the sensor and $\varepsilon$ is the signal noise. So, after acquiring $n$ voltage and temperature samples, we calculated regression coefficients in equation (1) by simple least square errors estimation, assuming Gauss-Markov conditions for $\varepsilon\left(E\left\{\varepsilon_{i}\right\}=0\right.$ and $\operatorname{Var}\left\{\varepsilon_{i}\right\}=\sigma_{i}^{2}$ ).

\subsection{In-use calibration procedure}

A healthy male subject was fitted with the IMU strapped to the (right) foot instep (Fig. 3). He was asked to perform a series of six movements. Each movement consisted of climbing one obstacle of given height, and it was performed at self-selected velocity. The gyroscope offset was estimated when the subject stood still before stepping. After subtracting the gyroscope offset, calculated as mean value of output voltage in steady conditions, from the gyroscope signal, the trajectory of the foot instep was reconstructed by strapdown integration; finally, the estimated vertical displacement was compared with the "true" value for a guessed value of the gyroscope sensitivity. The gyroscope sensitivity, which turned into the closest agreement between the estimated and the "true" vertical displacement was computed by averaging the six sensitivity values available from each stepping. In order to avoid over-fitting, the termination criterion of the optimization procedure was when the averaged estimation error was less than $2 \mathrm{~cm}$. Additionally, the whole procedure was repeated by submitting the sensory data to time-decimation before performing strapdown integration.
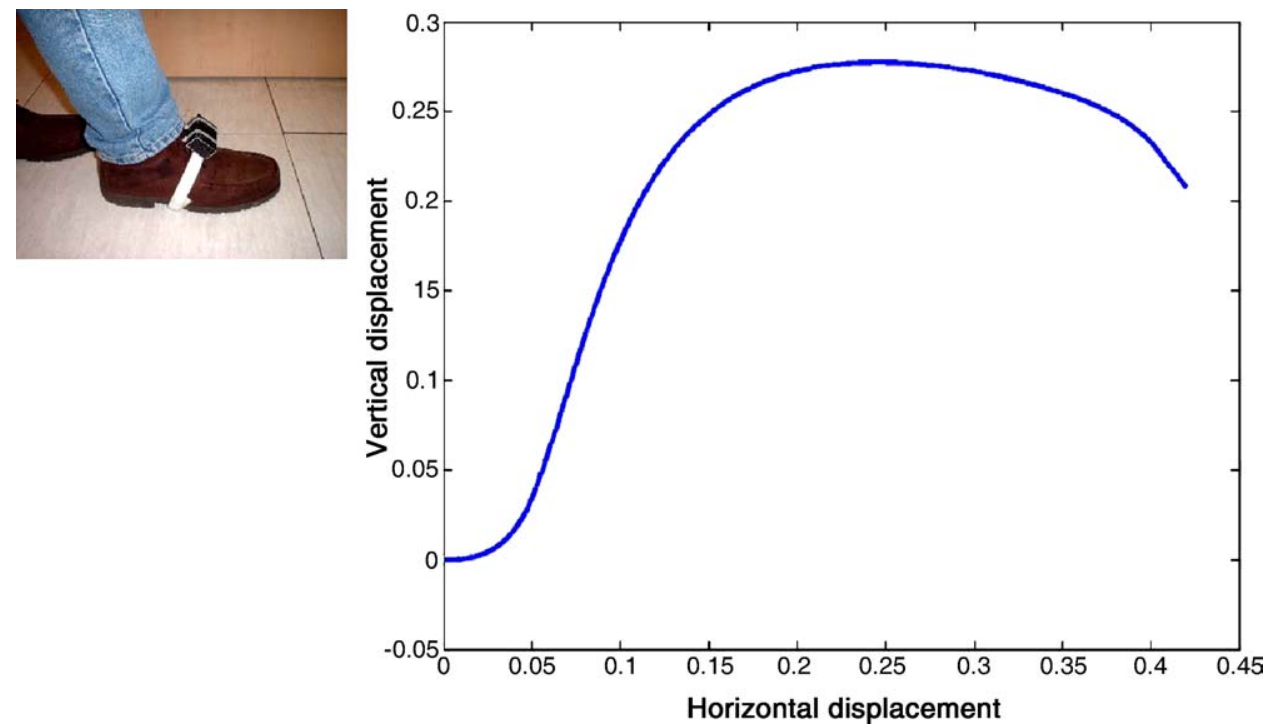

Fig. 3. Reconstructed trajectory of the foot instep. 
To verify calibration data obtained with this new procedure a complex task of human motion assessment was performed: it consists of five steps (step is $30 \mathrm{~cm}$ in length and $16 \mathrm{~cm}$ in height) stair climbed by the subject with IMU attached on his right foot instep. The subject starts at a distance of about $30 \mathrm{~cm}$ from first step and then climbs the stair at a self-selected velocity.

\section{Results}

The values of the offset off $\mathrm{a}_{\mathrm{a}}$ and sensitivity $K_{\mathrm{a}}$ of the two biaxial accelerometers integrated in the IMU are reported in Table 1. These values confirm the manufacturer specifications, which yield offsets within the interval $[-100,100] \mathrm{mV}$, and sensitivities within the interval $[85,125] \mathrm{mV} / \mathrm{g}$.

To evaluate regression coefficients of equation (1) we acquired data from RTD and gyroscope during $13 \mathrm{~h}$ at a sampling frequency of $0.05 \mathrm{~Hz}$ with temperature changing from 22 to $24^{\circ} \mathrm{C}$. The values founded are $\beta_{1}=0.306^{\circ} /\left(\mathrm{s}^{\circ} \mathrm{C}\right)$ and $\beta_{0}=1.310 \mathrm{~V}$, which corresponds to gyroscope offset in steady conditions at a temperature of about $23^{\circ} \mathrm{C}$.

Based on the results of the standard calibration procedure, the gyroscope sensitivity turns out to be $S_{\mathrm{V}}=$ $0.68 \mathrm{mV} /\left(^{\circ} \mathrm{s}\right.$ ) (the mean value of estimated rotation angle is $\theta=90.04^{\circ}$ )- the nominal sensitivity is $S_{\mathrm{V}}=0.67 \pm$ $20 \% \mathrm{mV} /\left({ }^{\circ} \mathrm{s}\right)$.

The trajectory of the sensed anatomical point - the foot instep - is reconstructed in the sagittal plane, as shown in Fig. 3. Table 2 reports the values of the errors incurred in the estimation of the vertical displacement (the "true" height of the step is $20.20 \mathrm{~cm}$ ), and the corresponding computed gyroscope sensitivity.

During these experiments temperature changed within the range $23.238 \pm 0.003^{\circ} \mathrm{C}$, so, considering regression coefficient $\beta_{1}$ found before, temperature effect was negligible during whole calibration procedure.

The statistics of the estimation errors show that we can reduce the sampling rate down to $200 \mathrm{~Hz}$ without significant penalty in the quality of the strapdown integration process.

Table 1

Accelerometer offset and sensitivity, estimated by standard calibration procedure

\begin{tabular}{lrrrr}
\hline & \multicolumn{1}{c}{$a_{x 1}$} & \multicolumn{1}{c}{$a_{y 1}$} & \multicolumn{1}{c}{$a_{x 2}$} & \multicolumn{1}{c}{$a_{y 2}$} \\
\hline off $_{\mathrm{a}}(\mathrm{mV})$ & 47.0 & 31.8 & -2.4 & 11.3 \\
$K_{\mathrm{a}}(\mathrm{mV} / \mathrm{g})$ & 103.4 & 102.7 & 100.2 & 101.6 \\
\hline
\end{tabular}

Table 2

Summary of results achieved with the proposed in-use calibration procedure

\begin{tabular}{lll}
\hline$f_{\mathrm{s}}(\mathrm{Hz})$ & Estimation error $(\mathrm{cm})$ & $S_{\mathrm{V}}\left(\mathrm{mV} /\left(^{\circ} \mathrm{s}\right)\right)$ \\
\hline 1000 & $0.36 \pm 1.24$ & 0.68 \\
500 & $0.47 \pm 1.27$ & 0.68 \\
200 & $0.50 \pm 1.39$ & 0.68 \\
100 & $0.60 \pm 1.93$ & 0.68 \\
\hline
\end{tabular}

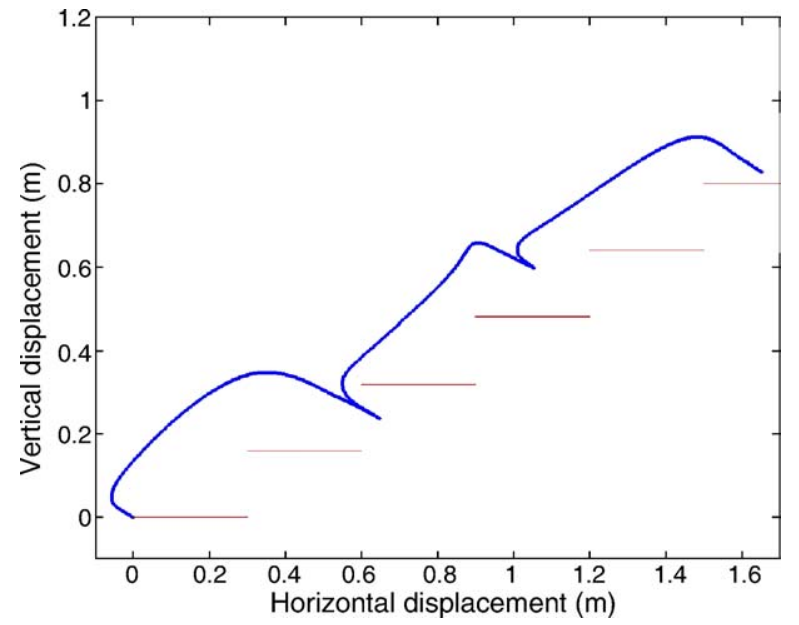

Fig. 4. Reconstructed trajectory of foot instep during stair climbing task, red lines correspond to real steps.

The estimated sensitivity $S_{\mathrm{V}}$ is equal to that computed by the standard calibration procedure at each tested sampling frequency. The stability of temperature, if we consider $\beta_{1}$ founded before, during both standard and in-use procedures assures reliability of results.

The presence of a small systematic error in the estimation of the vertical displacement does not preclude us from achieving success in the gyroscope calibration. That error can be attributed to three factors: use of cumulative integral via trapezoidal method for both accelerometric and gyroscopic signals, approximation of foot movement in a bidimensional space and electrical noise linked to sensors driver circuits.

The trajectory of foot instep during stair climbing task is shown in Fig. 4.

The real vertical and horizontal displacements covered by subject are, respectively, $0.80 \mathrm{~m}$ and about $1.80 \mathrm{~m}$ while mean values of those estimated using IMU are 0.84 and $1.68 \mathrm{~m}$, at a sampling frequency of $1 \mathrm{kHz}$. Less measurement error are on vertical displacement estimation, because it is fixed while the horizontal can vary based on foot final position on last step.

Considering real stair inclination $\left(\operatorname{arctg}(16 / 30)=28.07^{\circ}\right)$ and comparing it with the estimated one $(\operatorname{arctg}(0.84 / 1.68)=$ $26.60^{\circ}$ ) it was found an error of about $5 \%$ that is acceptable if compared with similar results obtained using inertial sensors for motion analysis reconstruction.

\section{Conclusions}

In this paper, we have proposed an in-use calibration procedure for gyroscopes, which has been applied to a simple IMU developed in our laboratory.

The calibration procedure does not require any tool or instrument, rather it can be used during normal use of the IMU, namely when the IMU is affixed to the moving body. 
Since the IMU is capable of reconstructing the trajectory of the sensed anatomical point - the foot instep, in the present application - the displacements in the three-dimensional space can be estimated by the IMU and compared with their true values, which are assumed to be known.

One movement, which corresponds to known displacements consist of foot placements from the ground level on top of steps of known height (step climbing). Provided that the IMU accelerometers are calibrated separately by any standard calibration procedure, the value of the gyroscope sensitivity is computed by looking for the value, which turns into the minimal estimation error after strapdown integration is performed.

\section{Acknowledgments}

This work has been supported in parts by a grant from the Fondazione Cassa di Risparmio di Pisa, Italy (KidRollbot project) and by the Italian Ministry of University and Research.

\section{References}

[1] E. Nebot, H. Durrant-Whyte, Initial calibration and alignment of an inertial navigation, in: Proceedings of Fourth Annual Conference on Mechatronics and Machine Vision in Practice, Toowomba, Australia, September 23-25, 1997, pp. 175-180.

[2] R.E. Mayagoitia, A.V. Nene, P.H. Veltink, Accelerometer and rate gyroscopes measurement of kinematics: an inexpensive alternative to optical motion analysis systems, J. Biomech. 35 (2002) 537-542.

[3] C.V.C. Bouten, K.T.M. Koekkoek, M. Verduin, R. Kodde, J.D. Janssen, A triaxial accelerometer and portable data processing unit for the assessment of daily physical activity, IEEE Trans. Biomed. Eng. 44 (3) (1997) 136-147.

[4] F. Ferraris, U. Grimaldi, M. Parvis, Procedure for effortless in-field calibration of three-axis rate gyros and accelerometers, Sens. Mater. 7 (5) (1995) 311-330.

[5] J.C. Lötters, J. Schipper, P.H. Veltink, W. Olthuis, P. Bergveld, Procedure for in-use calibration of triaxial accelerometers in medical applications, Sens. Actuators A 68 (1998) 221-228.

[6] I. Pappas, T. Keller, M. Popovic, Experimental evaluation of the gyroscope sensor used in a new gait phase detection system, in: Proc. of the 4th Ann. Conf. Int. Functional Electrical Stimulation Society, No. 4, Sendai, Japan, August 23-27, 1999, pp. 143-146.

[7] Y.S. Suh, Attitude estimation using low cost accelerometer and gyroscope, in: Proceedings of the Seventh KORUS International Symposium, Ulsan, Korea, June 29-July 2, 2003, pp. 423-427.

\section{Biographies}

Sergio Scapellato received the doctoral degree in electronic engineering from the University of Pisa, Pisa, Italy, in 2002. From January to December 2003, he worked as researcher for Advanced Robotics Technology and Systems Laboratory (ARTS Lab) of the Scuola Superiore Sant'Anna in Pontedera (PI), Italy, in 'Sensors, Signals and Systems for Rehabilitation' Group. Since January 2004, he is $\mathrm{PhD}$ student in bioengineering at the Scuola Superiore Sant'Anna, his research activity involves sensor network development and applications, motion analysis, inertial sensors calibration and biomedical signals processing.

Filippo Cavallo received the doctoral degree in electronic engineering at University of Pisa, Pisa, Italy, on March 2003. From April to December 2003, he worked in 'Sensors, Systems and Signals for Rehabilitation' Group. Currently, he is a PhD student in bioengineering at the Scuola Superiore Sant'Anna, Pisa, Italy, and his main topics are wearable sensor systems for rehabilitation, inertial sensors and GPS receivers for gait analysis and pedestrian applications, on-line DSP applications for standalone devices.

Chiara Martelloni received the doctoral degree biomedical engineering at the University of Pisa, Pisa, Italy, in 2002. Since 2002, she worked at the Advanced Robotics Technology and Systems Laboratory (ARTS Lab) of the Scuola Superiore Sant'Anna where she joined the "Sensors, Signals and Systems for Rehabilitation' Group. Her research interests include, ambulatory measurements, inertial sensing, gait analysis, calibration and signal processing.

Angelo M. Sabatini received the doctoral degree in electrical engineering in 1986 from the University of Pisa, Pisa, Italy, and the PhD degree in biomedical robotics in 1992 from Scuola Superiore Sant'Anna, Pisa. From 1987 to 1988, he was associated with the Centro E. Piaggio, Faculty of Engineering, University of Pisa. During the summer of 1988, he was a visiting scientist at the Artificial Organ Laboratory, Brown University, Providence, RI. Currently, he is an assistant professor of biomedical engineering at the Scuola Superiore Sant' Anna. His main research interests concern sensory information and digital signal processing in the field of biomedical robotics (design of intelligent assistive systems) and human performance engineering (assessment of human sensorymotor control performance capacity). 